\title{
Clinical Trial Inspection
}

National Cancer Institute

\section{Source}

National Cancer Institute. Clinical Trial Inspection. NCI Thesaurus. Code C142448.

The act by a regulatory authority(ies) of conducting an official review of documents, facilities, records, and any other resources that are deemed by the authority(ies) to be related to the clinical trial and that may be located at the site of the trial, at the sponsor's and/or contract research org anization's (CRO's) facilities, or at other establishments deemed appropriate by the regulatory authority(ies). (ICH) 